\title{
Two repetition time saturation transfer (TwiST) with spill-over correction to measure creatine kinase reaction rates in human hearts
}

Michael Schärr ${ }^{*}$, Refaat E. Gabr², AbdEl-Monem M. El-Sharkawy ${ }^{1,3}$, Angela Steinberg ${ }^{4}$, Paul A. Bottomley ${ }^{1}$ and Robert G. Weiss ${ }^{1,4}$

\begin{abstract}
Background: Phosphorus saturation transfer (ST) magnetic resonance spectroscopy can measure the rate of ATP generated from phosphocreatine ( $\mathrm{PCr}$ ) via creatine kinase (CK) in the human heart. Recently, the triple-repetition time ST (TRiST) method was introduced to measure the CK pseudo-first-order rate constant $\mathrm{k}_{\mathrm{f}}$ in three acquisitions. In TRiST, the longitudinal relaxation time of PCr while ${ }$-ATP is saturated, $T_{1}{ }^{\prime}$, is measured for each subject, but suffers from low SNR because the PCr signal is reduced due to exchange with saturated $\gamma$-ATP, and the short repetition time of one of the acquisitions. Here, a two-repetition time ST (TwiST) method is presented. In TwiST,

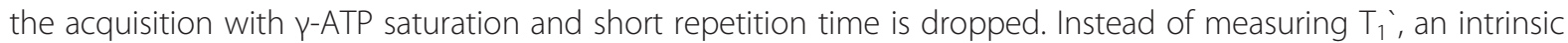
relaxation time $T_{1}$ for $P C r$, $T_{1}^{\text {intrinsic }}$, is assumed. The objective was to validate TwiST measurements of $C K$ kinetics in healthy subjects and patients with heart failure (HF).
\end{abstract}

Methods: Bloch equation simulations that included the effect of spillover irradiation on $\mathrm{PCr}$ were used to derive formulae for $T_{1}^{\text {intrinsic }}$ and $k_{f}$ measured by both TRiST and TwiST methods. Spillover was quantified from an unsaturated $\mathrm{PCr}$ measurement used in the current protocol for determining PCr and ATP concentrations. Cardiac TRiST and TwiST data were acquired at $3 \mathrm{~T}$ from 12 healthy and $17 \mathrm{HF}$ patients.

Results: Simulations showed that both $\mathrm{k}_{\mathrm{f}}$ measured by TwiST and $\mathrm{T}_{1}^{\text {intrinsic }}$ require spill-over corrections. In human heart at $3 \mathrm{~T}$, the spill-over corrected $T_{1}^{\text {intrinsic }}=8.4 \pm 1.4 \mathrm{~s}($ mean \pm SD) independent of study group. TwiST and TRiST $\mathrm{k}_{\mathrm{f}}$ measurements were the same, but TwiST was 9 min faster. Spill-over corrected TwiST $\mathrm{k}_{\mathrm{f}}$ was $0.33 \pm 0.08 \mathrm{~s}^{-1}$ vs. $0.20 \pm 0.06 \mathrm{~s}^{-1}$ in healthy vs HF hearts, respectively $(p<0.0001)$.

Conclusion: TwiST was validated against TRiST in the human heart at $3 \mathrm{~T}$, generating the same results 9 min faster. TwiST detected significant reductions in CK $\mathrm{k}_{\mathrm{f}}$ in HF compared to healthy subjects, consistent with prior $1.5 \mathrm{~T}$ studies using different methodology.

Keywords: Saturation transfer, Human heart, Metabolism, Reaction rate, High-energy phosphate, Heart failure, 3 Tesla, Creatine-kinase, TwiST

\footnotetext{
* Correspondence: michael.schar@gmail.com

${ }^{1}$ Division of MR Research, Russell H. Morgan Department of Radiology and Radiological Science, The Johns Hopkins University School of Medicine, Baltimore, MD, USA

Full list of author information is available at the end of the article
} 


\section{Background}

Phosphorus $\left({ }^{31} \mathrm{P}\right)$ saturation transfer $(\mathrm{ST})$ magnetic resonance spectroscopy (MRS) enables the in vivo study of adenosine triphosphate (ATP) kinetics including those through the creatine kinase (CK) reaction $[1,2]$. In muscle, the $\mathrm{CK}$ reaction serves as the prime energy reserve and a putative shuttle, transporting high-energy phosphates between the mitochondria, where ATP is created, and the myofibrils, where it is used. The pseudo-first-order rate constant $\left(\mathrm{k}_{\mathrm{f}}\right)$ for CK indexes the fractional rate of ATP generation from phosphocreatine (PCr). ${ }^{31} \mathrm{P}$ ST MRS using the Four Angle Saturation Transfer (FAST; [3]) protocol at $1.5 \mathrm{~T}$ enabled human cardiac CK kinetic studies for the first time and identified significant reductions in cardiac $\mathrm{k}_{\mathrm{f}}$ in patients with heart failure (HF). These findings and others indicated that reduced CK energy supply occurs in human HF, may play a role in the associated contractile dysfunction $[4,5]$, and is an independent predictor of subsequent clinical HF events [6].

In a ST experiment, the pseudo-first-order rate constant can be determined with:

$$
k_{f}=\frac{1}{T_{1}^{\prime}}\left(1-\frac{M_{0}{ }^{\prime}}{M_{0}}\right)
$$

where $\mathrm{T}_{1}{ }^{\prime}$ and $\mathrm{M}_{0}{ }^{\prime}$ are the longitudinal relaxation time and the equilibrium magnetization of $\mathrm{PCr}$ measured while the exchanging $\gamma$-ATP resonance at $-2.5 \mathrm{ppm}$ is selectively saturated (signified by primes); and $\mathrm{M}_{0}$ is the equilibrium magnetization of PCr measured without $\gamma-$ ATP saturation. Recently, the Triple Repetition time ST (TRiST) protocol was introduced to more efficiently measure $k_{f}$ in the human heart at $3 \mathrm{~T}$ [7]. TRiST only requires three acquisitions to determine $k_{\mathrm{f}}$. Each of the three acquisitions takes between 8 and $22 \mathrm{~min}$ as they employ chemical shift imaging (CSI) for localization and multiple averages for sufficient signal-to-noise ratio (SNR). Using TRiST, $\mathrm{T}_{1}{ }^{\prime}$ and $\mathrm{M}_{0}{ }^{\prime}$ are measured by the dual-repetition time (TR) method [8] with a short TR $\left(\mathrm{M}^{\prime}\left(\mathrm{TR}_{\text {short }}\right) ; \mathrm{TR}_{\text {short }}=2\right.$ heart beats, cardiac-gated $)$ and a long TR ( $\mathrm{M}^{`}$ ( $\left.\mathrm{TR}_{\text {long }}\right)$; $\mathrm{TR}_{\text {long }} \sim 10 \mathrm{~s}$, cardiac-gated) both while the exchanging $\gamma$-ATP resonance is saturated. A third cardiac-gated acquisition at a TR of $\sim 16 \mathrm{~s}$ is performed to measure $\mathrm{M}_{0}$. To compensate for the effects of spill-over irradiation on $\mathrm{PCr}$ during $\gamma$-ATP saturation, this third acquisition is performed while applying saturation at $+2.5 \mathrm{ppm}$-termed "control saturation", yielding $\mathrm{M}_{0}^{\text {control }}$. Equation [1] is then written as:

$$
k_{f}^{T R i S T}=\frac{1}{T_{1}{ }^{\prime}}\left(1-\frac{M_{0}{ }^{\prime}}{M_{0}^{\text {control }}}\right) .
$$

Spill-over irradiation is caused by imperfect frequencyselective saturation of $\gamma$-ATP that can partially saturate the nearby $\mathrm{PCr}$ resonance. A measure of the spill-over effect is provided by the ratio

$$
Q=\frac{M_{0}^{\text {control }}}{M_{0}}
$$

of the PCr signal acquired with control saturation, to the PCr signal acquired without any saturation $[3,9]$. The control saturation experiment does not fully compensate for the effect of spill-over on the observed $k_{f}$, and several methods have been presented to correct for the residual errors $[9,10]$. Nevertheless, the rate constant measured with TRiST appears relatively robust to varying levels of spill-over, as evidenced, for example, by essentially constant leg $\mathrm{k}_{\mathrm{f}}$ measurements at $3 \mathrm{~T}$ over regions wherein $Q$ varied from 0.5 to 0.9 [7].

The goal of the present work is to introduce and validate an even more efficient two-repetition time ST (TwiST) method for measuring $\mathrm{k}_{\mathrm{f}}$. TwiST is based on prior knowledge of the so-called "intrinsic $\mathrm{T}_{1}$ " of $\mathrm{PCr}$ $\left(T_{1}^{\text {intrinsic }}\right)$ which is independent of the chemical exchange processes implicit in Eqs [1] and [2]. The intrinsic $T_{1}$ was introduced long ago as the hypothetical $T_{1}$ that would occur if there were no chemical exchange, and is given by $[1,11]$ :

$$
T_{1}^{\text {intrinsic }}=T_{1}{ }^{\prime}\left(\frac{M_{0}^{\text {control }}}{M_{0}{ }^{\prime}}\right)
$$

If $\mathrm{T}_{1}^{\mathrm{intrinsic}}$ is known and is similar among groups studied, then $\mathrm{k}_{\mathrm{f}}$ is determinable from just two fully-relaxed measurements of $\mathrm{M}_{0}$ and $\mathrm{M}_{0}{ }^{\prime}[11,12]$. These two measurements comprise the TwiST experiment. In this case, $\mathrm{k}_{\mathrm{f}}$ is given by [13]:

$$
k_{f}^{T w i S T}=\frac{1}{T_{1}^{\text {intrinsic }}}\left(\frac{M_{0}^{\text {control }}}{M_{0}{ }^{\prime}}-1\right)
$$

The pre-requisite for performing TwiST is prior knowledge of $\mathrm{T}_{1}^{\text {intrinsic }}$. Here, $\mathrm{T}_{1}^{\text {intrinsic }}$ is determined from equation [4] and based on experimental data acquired from the hearts of healthy subjects and patients with HF. The effects of variations in the spill-over ratio, $Q$, on measurements of $\mathrm{T}_{1}^{\text {intrinsic }}, \mathrm{k}_{\mathrm{f}}^{\text {TRiST }}$, and $\mathbf{k}_{\mathrm{f}}^{\text {TwiST }}$ are evaluated by Bloch equation analysis. Spill-over correction for $\mathrm{T}_{1}^{\text {intrinsic }}, \mathrm{k}_{\mathrm{f}}^{\mathrm{TRiST}}$, and $\mathrm{k}_{\mathrm{f}}^{\mathrm{TwiST}}$ is derived analogous to the method described in [9]. If needed, this correction uses an unsaturated acquisition that is routinely recorded for determining $\mathrm{PCr}$ and ATP concentrations, and for measuring CK flux, $\left(\mathrm{k}_{\mathrm{f}} \mathrm{x}[\mathrm{PCr}]\right)$, in standard patient protocols $[4-6,14-18]$. 


\section{Methods}

Monte Carlo simulations without spill-over effects

Analogous to [7], Monte Carlo simulations were performed using Python 2.7 software (www.python.org) to determine the effect of low ${ }^{31} \mathrm{P}$ SNR on $\mathrm{k}_{\mathrm{f}}^{\text {TwiST }}$ for $0.1 \leq$ $\mathrm{k}_{\mathrm{f}} \leq 0.4 \mathrm{~s}^{-1}[4,5]$, and $5 \leq \mathrm{T}_{1}^{\text {intrinsic }} \leq 9 \mathrm{~s}$. These ranges were based on measured values for human heart, including an observed $\mathrm{T}_{1}$ of $\mathrm{PCr}, \mathrm{T}_{1, \mathrm{PCr}}$, of $5.8 \mathrm{~s}$ [8] which sets a lower limit to $\mathrm{T}_{1}^{\text {intrinsic }}$ because chemical exchange with ATP reduces $\mathrm{T}_{1, \mathrm{PCr}}$ [19]. Gaussian noise with a standard deviation (SD) of $\sigma=0.16 \mathrm{M}_{0}$ per acquisition was added 2000 times to the two TWiST acquisitions, $\mathrm{M}^{`}\left(\mathrm{TR}_{\mathrm{long}}\right)$ and $\mathrm{M}_{0}^{\text {control }}\left(\mathrm{TR}_{\text {control }}\right)$. $\mathrm{k}_{\mathrm{f}}^{\mathrm{TwiST}}$ was calculated from Eq. [5]. For estimating error, the SD of $\mathrm{k}_{\mathrm{f}}^{\mathrm{TwiST}}$ was calculated from the 2000 runs, and then averaged for the different $\mathrm{k}_{\mathrm{f}}$ and $\mathrm{T}_{1}^{\text {intrinsic }}$. Fifty-nine different combinations of averages and cardiac-gated TRs that resulted in a fixed total scan time of $\sim 30 \mathrm{~min}$ for the two acquisitions were evaluated. For each TR combination, the number of averages leading to the lowest error was selected. Bias was determined for each sequence combination with a mid-range $\mathrm{T}_{1}^{\text {intrinsic }}=7$ s only.

\section{Bloch equation calculation of spill-over effects}

In the prior TRiST protocol [7], a surface coil was used for RF transmission, producing an inhomogeneous excitation field with high intensity close to the coil and a gradual decline moving away from it. An adiabatic excitation pulse was used to generate a homogeneous flipangle (FA) over the region of interest, and a modulated DANTE pulse train was used for saturating the exchanging $\gamma$-ATP moiety. The DANTE pulses are subject to inhomogeneity in the transmit field, and were set to provide sufficient saturation at the depth of the heart. This causes spill-over saturation of $\mathrm{PCr}$ closer to the coil, which is quantified by the variable $Q$ in Eq. 3. Its effects on the measured $\mathrm{T}_{1}^{\text {intrinsic }}, \mathrm{k}_{\mathrm{f}}^{\mathrm{TRiST}}$ and $\mathrm{k}_{\mathrm{f}}^{\text {TwiST }}$ are determined here by numerical analysis of the Bloch-McConnell equations modified for two-site chemical exchange in matrix form $[3,20]$ implemented on a graphical programming interface (GPI) [21].

The PCr signals, $M_{0}(T R=16 \mathrm{~s})$, without any saturation; $\mathrm{M}_{0}^{\text {control }}(\mathrm{TR}=16 \mathrm{~s})$ with control saturation at $+130 \mathrm{~Hz}$; and $\mathrm{M}_{\mathrm{PCr}}^{\prime}(\mathrm{TR}=10 \mathrm{~s})$ and $\mathrm{M}_{\mathrm{PCr}}^{\prime}(\mathrm{TR}=1.7 \mathrm{~s})$ with $\gamma$-ATP saturated at $-130 \mathrm{~Hz}$, were all determined for the TRiST and TwiST experiments. The saturation of $\gamma$-ATP by the amplitude-modulated DANTE scheme described in Eq. 5 of [7] was simulated with parameters used in human studies $(\mathrm{m}=5$ suppression bands; $\delta=9 \mathrm{~Hz}$ separation between bands; $\beta=0.9^{\circ}$ FA per band as expected $10 \mathrm{~cm}$ from the coil [8]; $\tau=0.91 \mathrm{~ms}$ between hard sub-pulses of $100-\mu$ s duration).

To simulate a range of spill-over strengths, $\beta$ was varied from $0.1^{\circ}$ to $6.0^{\circ}$. Other parameters were: $\mathrm{T}_{1}^{\text {intrinsic }}=$
$7900 \mathrm{~ms}$; $\mathrm{T}_{1, \mathrm{ATP}}^{\text {intrinsic }}=2200 \mathrm{~ms}$ for the $\mathrm{T}_{1}^{\text {intrinsic }}$ of ATP; with corresponding spin-spin relaxation times $\left(\mathrm{T}_{2}\right), \mathrm{T}_{2, \mathrm{PCr}}=$ $250 \mathrm{~ms}$ and $\mathrm{T}_{2, \mathrm{ATP}}=50 \mathrm{~ms}$ chosen somewhat shorter than values measured in calf muscle [22]. To simulate the effect of static magnetic field inhomogeneity, the calculations were performed 9 times with the saturation frequency offset by -20 to $+20 \mathrm{~Hz}$ in steps of $5 \mathrm{~Hz}$. Results from the 9 runs were weighted with a $20 \mathrm{~Hz}$ full-width-half-maximum Gaussian function and averaged. Based on the four calculated $M_{0}$ and $M^{`} \mathrm{PCr}$ signals, simulated values of $\mathrm{T}_{1}^{\text {intrinsic }}, \mathrm{k}_{\mathrm{f}}^{\mathrm{TR}} \mathrm{iST}$, and $\mathrm{k}_{\mathrm{f}}^{\text {TwiST }}$ were determined from equations [4], [2], and [5], respectively. Two different "true" $\mathrm{k}_{\mathrm{f}} \mathrm{s}$ of $0.21 \mathrm{~s}^{-1}$ and $0.32 \mathrm{~s}^{-1}$ were assumed, reflecting previous rates measured at $1.5 \mathrm{~T}$ for $\mathrm{HF}$ patients and healthy subjects, respectively [4].

\section{Spill-over corrections for intrinsic $\mathrm{T}_{1}, \mathrm{TRiST}$ and TwiST}

Spill-over corrected $\mathrm{T}_{1}^{\text {intrinsic }}$, TRiST and TwiST formulae were determined using the approach of Gabr et al. [9]. The Bloch-McConnell equations [20] were numerically solved for the range of parameters listed in Table 1, with the range of saturation power limited to $\mathrm{Q} \leq 0.96$ to ensure sufficient saturation of $\gamma$-ATP (see Discussion). A minimum sum-of-the-squared fractional differences algorithm was applied to fit the calculated data to linear spill-over corrected formulae with an affine dependence on the measured parameters. The corrected intrinsic $\mathrm{T}_{1}, \mathrm{~T}_{1}^{\mathrm{Q}-\text { intrinsic }}$, had the form:

$$
T_{1}^{Q-\text { intrinsic }}=a\left(\frac{M_{0}^{\prime}}{M_{0}^{\text {control }}}+b\right)(Q+c)\left(T_{1}^{\prime}+d\right)
$$

The Q-corrected TRiST, $\mathrm{k}_{\mathrm{f}}^{\mathrm{Q}-\mathrm{TRiST}}$ was:

$$
k_{f}^{Q-T R i S T}=f\left(\frac{M_{0}^{\prime}}{M_{0}^{\text {control }}}+g\right)(Q+h)\left(T_{1}^{\prime}+k\right) .
$$

And the Q-corrected TwiST, $\mathrm{k}_{\mathrm{f}}^{\mathrm{Q}-\mathrm{TwiST}}$ was formulated as:

Table 1 Parameter ranges of the two exchanging metabolites of the CK reaction used to determine the spill-over corrected formulae for cardiac TRiST and TwiST at 3 Tesla

\begin{tabular}{llll}
\hline Parameter & Min & Max & Fixed \\
\hline PCr/ATP & 1 & 2 & \\
intrinsic $\mathrm{T}_{1, \mathrm{PCr}}[\mathrm{s}]$ & 6.5 & 9.5 & \\
intrinsic $\mathrm{T}_{1, \mathrm{Y} \text {-ATP }[\mathrm{s}]}$ & 2 & 3.5 & \\
$\mathrm{~T}_{2, \mathrm{PCr}}[\mathrm{s}]$ & & & 0.2 \\
$\mathrm{~T}_{2, \mathrm{r}^{-A T P}[\mathrm{~s}]}$ & & & 0.05 \\
$\mathrm{k}_{\mathrm{f}}\left[\mathrm{s}^{-1}\right]$ & 0.1 & 0.4 & \\
DANTE $\beta\left[^{\circ}\right]$ & 0.8 & 4.0 & \\
$\Delta \mathrm{f}[\mathrm{Hz}]$ & & & 130 \\
\hline
\end{tabular}




$$
k_{f}^{Q-T w i S T}=l\left(\frac{M_{0}^{\text {control }}}{M^{\prime}\left(T R_{\text {long }}\right)}+m\right)(Q+n)
$$

where a-n are fitting coefficients.

These new spill-over corrected formulae were applied to the simulated $\mathrm{PCr}$ signals generated from the previous section.

\section{Human studies}

Human studies were approved by the Institutional Review Board of the Johns Hopkins University School of Medicine, with all participants providing written informed consent. TRiST data, that included TwiST data as a subset, were acquired on a $3 \mathrm{~T}$ broadband Achieva scanner (Philips Healthcare, Best, the Netherlands) from twelve healthy subjects ( 7 men, 5 women, mean age of $36 \pm 15$ years) with no history of hypertension, diabetes, or heart disease; and in seventeen HF patients (8 men, 9 women, mean age of $48 \pm 15$ years) with a clinical history of HF (New York Heart Association class I (2), class II (8), and class III (7)), a left ventricular ejection fraction $<40 \%$, and no significant coronary disease.

Both TwiST and reference TRiST data were acquired from a single TRiST protocol described in [7]. Guided by scout MRI, participants were oriented prone with the heart centered above a custom built ${ }^{31} \mathrm{P}$ coil set with dual loop 17/11-cm diameter transmit and $8-\mathrm{cm}$ receive coils [8] that had a fiducial marker at its center (Fig. 1). Localized $2^{\text {nd }}$-order shimming was performed based on acquired field mapping [23]. Axial balanced steady-state free precession cine images were acquired during free breathing to determine the trigger delay for MRS acquisition at end-systole, which was chosen to minimize motion and maximize the amount of cardiac tissue close to the coil. Cardiac-triggered, 1D CSI data were acquired with sixteen phase encodes from a 16-cm field-of-view using frequency-sweep-cycled adiabatic half-passage [8] excitation. A first data set was acquired without saturation at $T R \geq 16 \mathrm{~s}$ and 2 averages to measure $\mathrm{M}_{0}$. This data set was used to center the saturation frequency on the cardiac $\gamma$-ATP resonance and to determine the spill-over ratio Q; it can also be used to determine metabolite concentrations [14] (not reported here). Next, three TRiST data sets were acquired: the first, $\mathrm{M}_{0}^{\text {control }}$, with control saturation applied $\left(\mathrm{TR}_{\text {control }} \geq 16 \mathrm{~s} ; 2\right.$ averages); the second, $\mathrm{M}^{\prime}\left(\mathrm{TR}_{\text {long }}\right)$, with $\gamma$-ATP saturated ( $\mathrm{TR}_{\text {long }} \geq 10 \mathrm{~s} ; 8$ averages); and the third, $\mathrm{M}^{`}\left(\mathrm{TR}_{\text {short }}\right)$ also with $\gamma$-ATP saturated $\left(\mathrm{TR}_{\text {short }}=2\right.$ heart beats; 18 averages). The third acquisition was not used in the TwiST analysis. The average TR of each triggered acquisition was determined from the scanner's physiological log.

Spectra from the anterior myocardium were analyzed as described in [7]. A semi-automatic tool (IDL 6.3, Exelis Visual Information Solutions, Boulder, Colorado) was used to measure $\mathrm{PCr}$ signals from peak heights after subtracting the baseline (Fig. 2). Peak height instead of area is used because signal ratios are determined from acquisitions with identical shim settings. User interactions were limited to the selection of cardiac slices (including quarter or half slice Fourier shift) and zero order phasing. Identical phasing is used for all four acquisitions. The baseline is

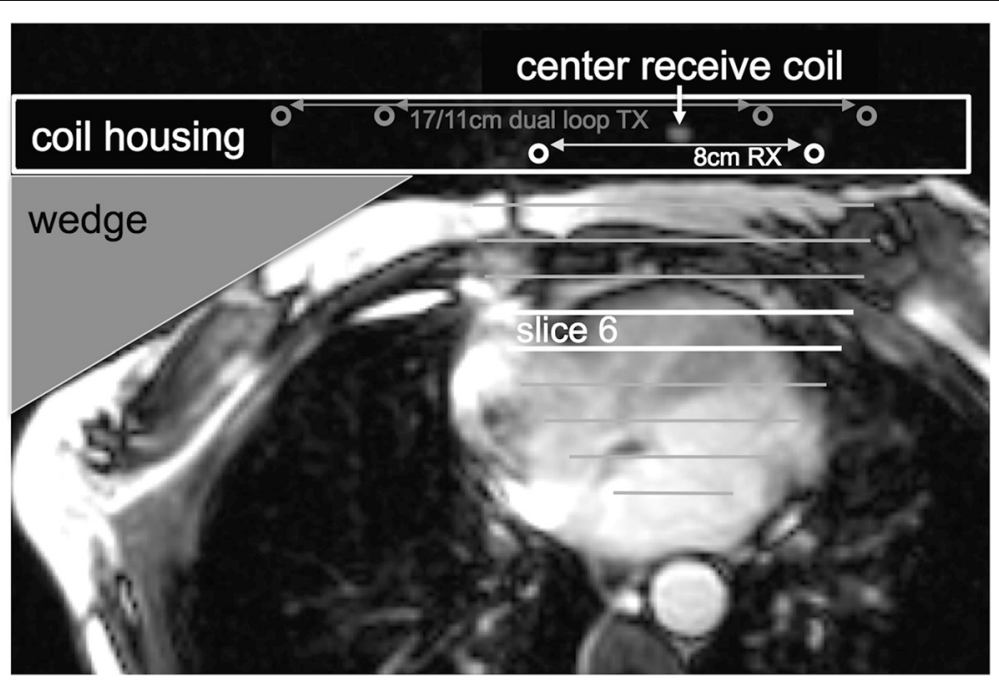

Fig. 1 Axial, end-systolic time frame of a balanced steady-state free precession cine image illustrating the patient setup. Participants were oriented prone on the coil which includes a marker at the center of the receive coil. Circles depict location of $8 \mathrm{~cm}$ diameter receive (white) and $17 / 11 \mathrm{~cm}$ diameter dual loop transmit coil (gray) conductors. A wedge is used to rotate the participant to ensure the center of the receive coil is straight in front of the cardiac septum. Lines indicate the slices of 1-dimensional CSI localization combined with receive coil sensitivity. Bright lines indicate the $6^{\text {th }}$ slice, the origin of example spectra shown in Fig. 2 

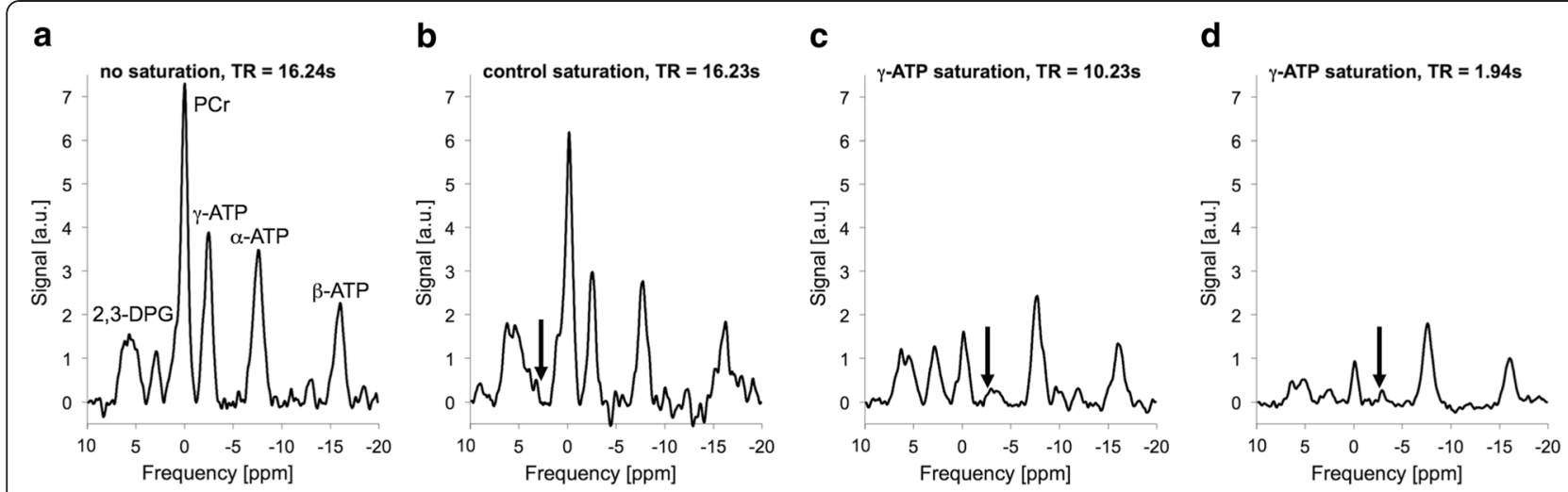

Fig. 2 Example cardiac spectra acquired from the $6^{\text {th }}$ slice of 1DCSI setup illustrated in Fig. 1. a 9 min acquisition without any saturation used to determine $\mathrm{M}_{0}$ from the peak height of the $\mathrm{PCr}$ peak. This spectrum is also used to determine the saturation frequency of the $\gamma$-ATP peak in the heart. b 9 min acquisition with control saturation at the frequency indicated by the black arrow used to determine $\mathrm{M}_{0}^{\text {control }}$. c 22 min acquisition with $\gamma$-ATP saturation (black arrow) and long TR used to determine $M_{0}{ }^{\prime}$ in the TwiST experiment, and $M^{\prime}$ (TR I $_{\text {long }}$ ) in the TRiST experiment. $\mathbf{d}$ This 9 min acquisition is only used in TRiST but not in TwiST. It is acquired with $\gamma$-ATP saturation (black arrow) and short TR and used to determine

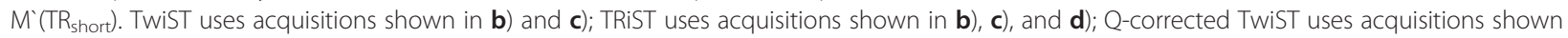
in $\mathbf{a}), \mathbf{b})$, and $\mathbf{c}$ ); and Q-corrected TRiST uses acquisitions shown in $\mathbf{a}), \mathbf{b}), \mathbf{c}$ ), and $\mathbf{d}$ )

determined automatically by averaging values around the minimum points of the peak of interest. $\mathrm{T}_{1}{ }^{`}$ and $M_{0}{ }^{\prime}$ were determined from $M^{\prime}\left(T R_{\text {short }}\right)$ and $M^{\prime}\left(T R_{\text {long }}\right)$ using the dual-TR method [8]. To perform spill-over corrections, Q was determined for all cardiac spectra using Eq. [3]. Q values larger than one (due to SNR fluctuations) were set equal to 1.0.

$\mathrm{T}_{1}^{\text {intrinsic }}$ and spill-over corrected $\mathrm{T}_{1}^{\mathrm{Q}-\text {-intrinsic }}$ were calculated for each cardiac slice using Eq. [4] and [6], respectively, and averaged for each participant. The mean and $\mathrm{SD}$ of $\mathrm{T}_{1}^{\text {intrinsic }}$ and $\mathrm{T}_{1}^{\mathrm{Q} \text {-intrinsic }}$ were determined for the healthy and the HF groups, and the two groups compared using an unpaired Student's $t$-test. Uncorrected and $\mathrm{Q}$-corrected intrinsic $\mathrm{T}_{1}$ values were compared with a paired Student's $t$-test.

$k_{f}^{\text {TRiST }}$ and $k_{f}^{\text {TwiST }}$ were determined for all cardiac slices using Eq. [2] and [5], respectively. For $\mathrm{k}_{\mathrm{f}}^{\mathrm{TwiST}}, \mathrm{T}_{1}^{\text {intrinsic }}=$ $7.9 \mathrm{~s}$ was used. Spill-over corrected $\mathrm{k}_{\mathrm{f}}^{\mathrm{Q}-\mathrm{TR} \text { iST }}$ and $\mathrm{k}_{\mathrm{f}}^{\mathrm{Q}-\mathrm{TwiST}}$ were determined using Eq. [7] and [8]. All cardiac values were averaged for each participant. TRiST and TwiST $k_{f}$ values were compared with and without $\mathrm{Q}$-corrections using paired Student's t-testing, linear regression, and Bland-Altman analysis. The mean and SD of Qcorrected $\mathrm{k}_{\mathrm{f}}^{\mathrm{Q}-\mathrm{TwiST}}$ were determined for the healthy and HF groups, and the two cohorts compared by unpaired Student's t-testing. A $p<0.05$ was considered significant for all statistical testing.

\section{Results}

\section{Simulations}

Figure 3 shows the results of the Monte Carlo simulations without spill-over effects. Figure $3 \mathrm{a}$ and $\mathrm{b}$ can be compared directly to that of TRiST in Fig. 3 of [7]. The average SD (Fig. 3a) of TwiST is lower and varies less for different TRs than the TRiST result [7]. The expected noise-induced SD at the TRs used in the present study is $8.3 \%$ compared to $13.4 \%$ for TRiST [7]. Unlike TRiST, the bias error in TwiST (Fig. 3b) depends strongly on $\mathrm{TR}_{\text {long. For }} \mathrm{TR}_{\text {long }}<8 \mathrm{~s}$ the negative bias error grows rapidly. The currently applied $\mathrm{TR}_{\text {long }}$ of $10 \mathrm{~s}$ is in the flat part of the graph and therefore a reasonable

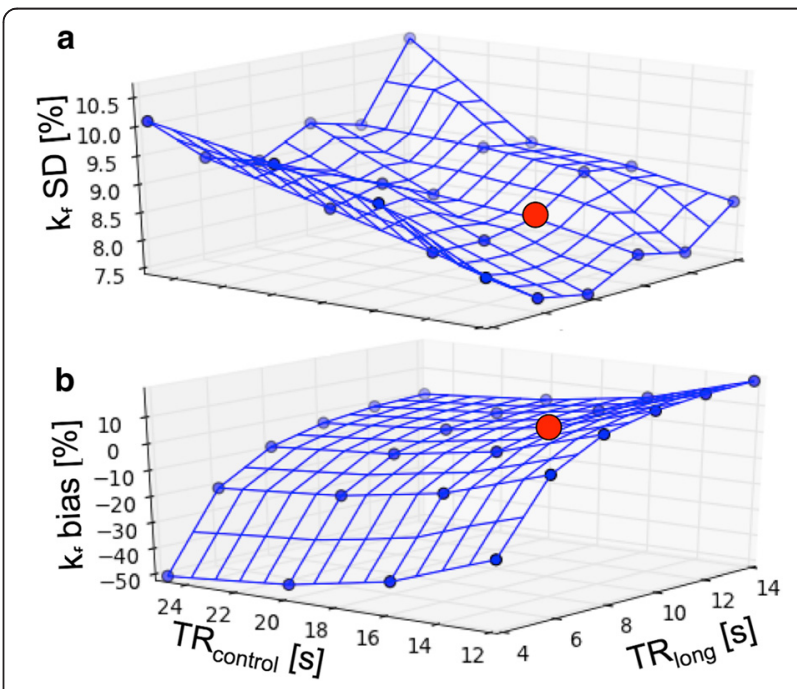

Fig. 3 Results from Monte Carlo simulations of the TwiST experiment (without spill-over correction) over a range of $\mathrm{TR}_{\mathrm{long}}$ (with $\mathrm{Y}$-ATP saturated) and $\mathrm{TR}_{\text {control }}$ (control saturation), averaged over a range of $\mathrm{k}_{\mathrm{f}}$ from $0.1-0.4 \mathrm{~s}^{-1}$ and $\mathrm{T}_{1}^{\mathrm{intrinsic}}$ from 5-9 $\mathrm{s}$. Averages were chosen for a constant total study time of $30 \mathrm{~min}$. a The relative percentage SD in $\mathrm{k}_{f}$ for average choices that led to the smallest error at each TR combination. $\mathbf{b}$ The average bias error in $\mathrm{k}_{\mathrm{f}}$ corresponding to the same TR and average combinations as in a) and $\mathrm{T}_{1}^{\text {intrinsic }}$ of $7 \mathrm{~s}$. The red dot illustrates the TRs used in the human acquisitions in this study $\left(\mathrm{TR}_{\text {long }}=10 \mathrm{~s}\right.$ and $\left.\mathrm{TR}_{\text {control }}=16 \mathrm{~s}\right)$ 
choice. In this regime, the bias error increases slightly when decreasing $\mathrm{TR}_{\text {control}}$, and $\mathrm{TR}_{\text {control }}=16 \mathrm{~s}$ remains a reasonable choice.

The effects of spill-over as determined by the Bloch equation simulations are shown in Fig. 4. The $T_{1}^{\text {intrinsic }}$ (Fig. 4a and b, blue lines) determined in the presence of spillover underestimates the true value in proportion to the amount of spillover, as indexed by declining $\mathrm{Q}$. The underestimation of $T_{1}^{\text {intrinsic }}$ is independent of $\mathrm{k}_{\mathrm{f}}$ at $0.21 \mathrm{~s}^{-1}$ (Fig. 4a) vs. $0.32 \mathrm{~s}^{-1}$ (Fig. 4b). Figure $4 \mathrm{c}$ and $\mathrm{d}$ show the deviation in $\mathrm{k}_{\mathrm{f}}^{\mathrm{TR}}$ iST (blue lines) from the true values (black, dashed lines) vs. Q for the TRiST method. The error stays within about $10 \%$ for $\mathrm{Q}>0.6$ but increases with higher spill-over $(\mathrm{Q}<0.6)$. For the TwiST method, $\mathrm{k}_{\mathrm{f}}^{\text {TwiST }}$ varies more strongly with $\mathrm{Q}$ (blue lines in Fig. 4e and $\mathrm{f}$ ). At high levels of spill-over $(\mathrm{Q} \sim 0.4), \mathrm{k}_{\mathrm{f}}^{\text {TwiST }}$ is underestimated by $\sim 50 \%$, and a spill-over correction is required over most of the range.

The coefficients of the spill-over corrected formulas for $\mathrm{T}_{1}^{\mathrm{Q} \text {-intrinsic }}$ (Eq. [6]), $\mathrm{k}_{\mathrm{f}}^{\mathrm{Q}-T R i S T}$ (Eq. [7]) and $\mathrm{k}_{\mathrm{f}}^{\mathrm{Q}-T w i S T}$ (Eq. [8]) are listed in Table 2. The calculated relative errors of $T_{1}^{\text {intrin- }}$ sic, $k_{f}^{\text {TRiST }}$ and $k_{f}^{\text {TwiST }}$ over the simulated range of parameters (Table 1) before and after spill-over correction are shown in Table 3. Spill-over correction removes the bias
Table 2 Coefficients for spill-over corrected $T_{1}^{\text {Q-intrinsic }}$ (Eq. [6]), $k_{f}^{Q-T R i S T}$ (Eq. [7]) and $k_{f}^{\mathrm{Q}-T w i S T}$ (Eq. [8]) were calculated for cardiac CK exchange measured with modulated DANTE saturation as described in [7] at 3 Tesla for a parameter range given in Table 1

\begin{tabular}{lllll}
\hline Formula & \multicolumn{4}{c}{ Coefficients } \\
\hline$T_{1}^{Q-\text {-intrinsic }}$ & $\mathrm{a}=4.2803$ & $\mathrm{~b}=-1.1344$ & $\mathrm{c}=-1.6610$ & $\mathrm{~d}=0.7862$ \\
$\mathrm{k}_{\mathrm{f}}^{\mathrm{Q}-\text { TRisT }}$ & $\mathrm{f}=0.0052$ & $\mathrm{~g}=-0.8730$ & $\mathrm{~h}=27.5332$ & $\mathrm{k}=-6.0647$ \\
$\mathrm{k}_{\mathrm{f}}^{\mathrm{Q}-\text { TwiST }}$ & $\mathrm{l}=-0.2013$ & $\mathrm{~m}=-1.0305$ & $\mathrm{n}=-1.6113$ & \\
\hline
\end{tabular}

error and moderately reduces the error range, which includes the effect of a varying $T_{1}^{\text {intrinsic }}$ in accordance with Eq. 5. The relative error of $\mathrm{k}_{\mathrm{f}}^{\mathrm{Q}-T w i S T}$ for the same parameter range is plotted in Fig. 5 versus the $T_{1}^{\text {intrinsic }}$ used in the simulations. As expected, the bias error for $\mathrm{T}_{1}^{\text {intrinsic }}$ outside of $8-8.5 \mathrm{~s}$ is proportional to the actual $\mathrm{T}_{1}^{\text {intrinsic }}$. Applied to the simulations shown in Fig. 4, the spill-over correction (red lines) improves the determined intrinsic $\mathrm{T}_{1}$, (Fig. $4 \mathrm{a}$ and $\mathrm{b}$ ) and TwiST $k_{f}$ (Fig. 4e and f) for a wide range of $Q$, and TRiST $\mathrm{k}_{\mathrm{f}}$ for $\mathrm{Q}<0.6$ (Fig. 4c and d).

\section{Experiments}

The total acquisition time including repositioning and scout MRI at the beginning of each study to optimally

\section{a}

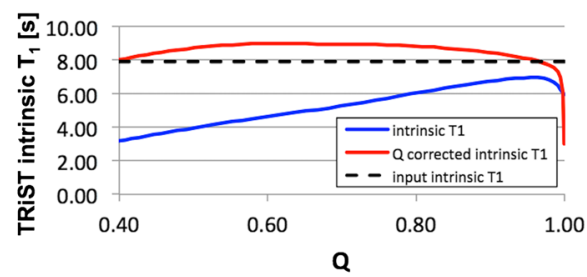

C

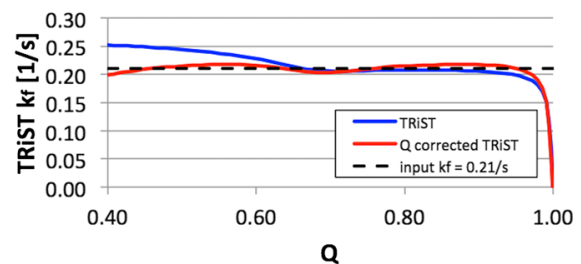

e

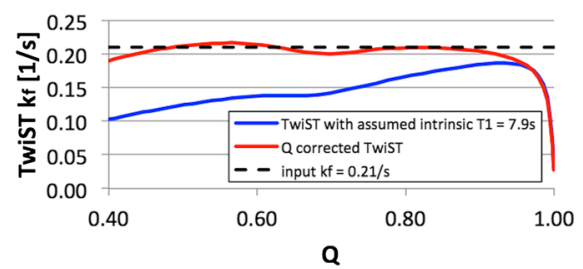

b

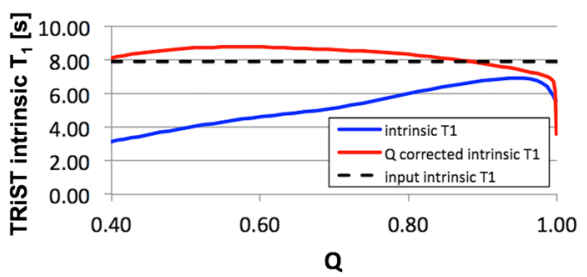

d

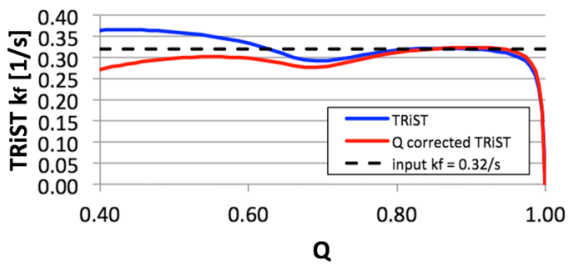

f

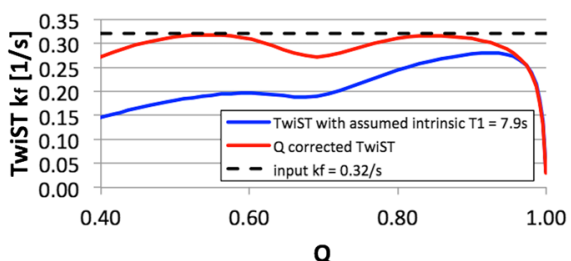

Fig. 4 Spill-over effects as determined by Bloch equation simulations: $T_{1}^{\text {intrinsic }}$ (solid blue line) as determined with the TRiST method and spill-over

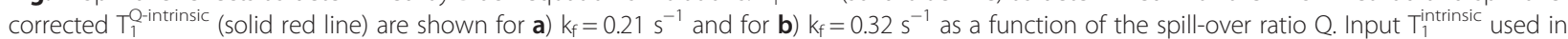
the simulation was $7.9 \mathrm{~s}$ (dotted black line). c) and $\mathbf{d}$ ) show the pseudo-first-order rate constant $\mathrm{k}_{\mathrm{f}}$ as determined with TRiST (solid blue line) and with Q-corrected TRiST (solid red line) for $k_{f}=0.21 \mathrm{~s}^{-1}$ and $k_{f}=0.32 \mathrm{~s}^{-1}$ (dotted black line), respectively. And $\mathrm{k}_{\mathrm{f}}$ determined with TwiST (solid blue line) and Q-corrected TwiST (solid red line) is shown e) for $k_{f}=0.21 \mathrm{~s}^{-1}$ and $\mathbf{f}$ ) for $\mathrm{k}_{\mathrm{f}}=0.32 \mathrm{~s}^{-1}$ (dotted black line). Note that the dip in $\mathrm{T}_{1}^{\text {intrinsic }}$ and $\mathrm{k}_{\mathrm{f}}$ for $\mathrm{Q}>0.95$ is caused by incomplete $\mathrm{Y}$-ATP saturation, as the range in $\mathrm{Q}$ is induced by the variation of the DANTE saturation flip angles 
Table 3 Simulated relative errors of $T_{1}^{\text {intrinsic }}, k_{f}^{\text {TRiST }}$ and $k_{f}^{\text {TwiST }}$ before and after Q spill-over correction determined over the range of parameters (Table 1) used to determine the coefficients for the spill-over corrected formulae (Table 2), including the effect of a varying $T_{1}^{\text {intrinsic }}$ per Eq. [5]

\begin{tabular}{lll}
\hline & $\begin{array}{l}\text { Relative error [\%] } \\
(\text { mean } \pm \text { SD) }\end{array}$ & $\begin{array}{l}\text { Relative error [\%] } \\
\text { range }(\mathrm{min}, \mathrm{max})\end{array}$ \\
\hline $\mathrm{T}_{1}^{\text {intrinsic }}$ (Eq. 4) & $-37.0 \pm 16.4$ & $(-66.2,-9.9)$ \\
$\mathrm{T}_{1}^{\mathrm{Q} \text {-intrinsic }}$ (Eq. 6) & $0.8 \pm 7.9$ & $(-23.2,16.2)$ \\
$\mathrm{k}_{f}^{\text {TRiST }}$ (Eq. 2) & $12.0 \pm 12.8$ & $(-8.8,71.8)$ \\
$\mathrm{k}_{\mathrm{f}}^{\mathrm{Q} \text { TRiST }}$ (Eq. 7) & $-1.1 \pm 10.3$ & $(-35.8,40.1)$ \\
$\mathrm{k}_{f}^{\text {TwiST }}$ (Eq. 5) & $-27.6 \pm 17.7$ & $(-60.6,27.6)$ \\
$\mathrm{k}_{f}^{\text {Q-TwiST }}$ (Eq. 8) & $-2.3 \pm 14.9$ & $(-36.8,47.3)$ \\
\hline
\end{tabular}

position the coil for cardiac TRiST was $84 \pm 10 \mathrm{~min}$ (mean \pm SD) for all participants. This included the $9 \mathrm{~min}$ to acquire the unsaturated $M_{0}$ data set with no saturation used for the Q corrections and metabolite quantification. The duration of the three TRiST acquisitions was $40 \pm 1 \mathrm{~min}$. Omitting the $\mathrm{M}^{\prime}\left(\mathrm{TR}_{\text {short }}\right)$ acquisition, which is no longer needed for TwiST, reduces the effective acquisition time by $9 \pm 1 \mathrm{~min}$, for a $23 \%$ efficiency improvement for TwiST.

Cardiac PCr $\mathrm{T}_{1}^{\text {intrinsic }}$ are shown in Fig. 6, both with and without $\mathrm{Q}$-correction. $\mathrm{T}_{1}^{\text {intrinsic }}$ with and without Q-correction does not differ significantly between patients and healthy controls. However, spill-over correction significantly increases the intrinsic $\mathrm{T}_{1}$. With Qcorrection, $\mathrm{T}_{1}^{\mathrm{Q}-i n t r i n s i c}=8.2 \pm 1.3 \mathrm{~s}$ in healthy subjects vs. $8.5 \pm 1.5 \mathrm{~s}$ in $\operatorname{HF}(p=0.6)$. The average spill-over

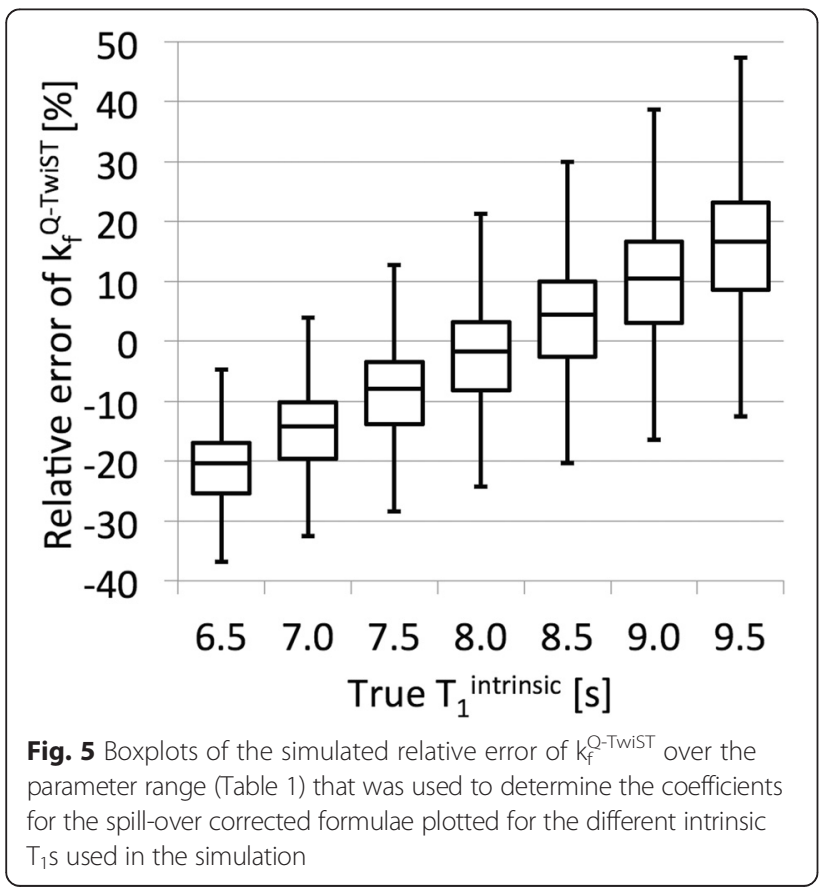

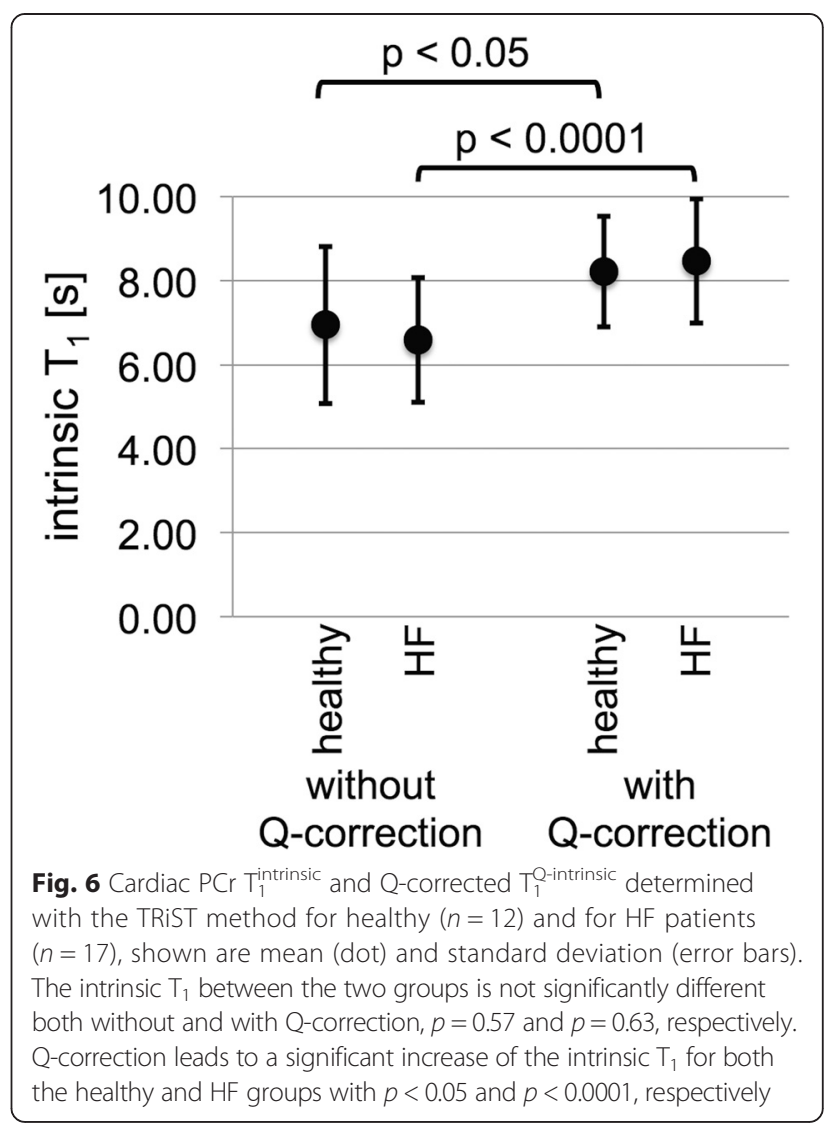

corrected $\mathrm{T}_{1}^{\mathrm{Q}-\text { intrinsic }}$ in all participants is $8.4 \pm 1.4 \mathrm{~s}$. The $Q$ values were the same in both groups at $0.84 \pm$ 0.13 in healthy subjects and $0.85 \pm 0.11$ in HF $(p=0.8)$.

TwiST $k_{f}$ measurements are compared to the previously validated TRiST $k_{f}$ in Fig. 7. Linear regression (Fig. 7a and b) reveals a significant correlation between $\mathrm{k}_{\mathrm{f}}^{\text {TRiST }}$ and $\mathrm{k}_{\mathrm{f}}^{\text {TwiST }}$ calculated both without $\left(\mathrm{R}^{2}=0.53\right)$ and with $\left(\mathrm{R}^{2}=0.73\right) \mathrm{Q}$-corrections $(p<0.0001$ for both). Corresponding Bland-Altman plots in Fig 7c and d show that the Q-correction reduces scatter.

Figure 8 presents box plots for $\mathrm{k}_{\mathrm{f}}$ determined with spill-over Q-corrected TRiST and TwiST methods for healthy and HF patients measured at $3 \mathrm{~T}$. $\mathrm{K}_{\mathrm{f}}^{\mathrm{Q}-\mathrm{T} w i S \mathrm{~T}}$ are the same as $\mathrm{k}_{\mathrm{f}}^{\mathrm{Q}-\mathrm{TRiST}}$ values for both healthy subjects $(p=0.2)$ and HF patients $(p=0.8)$. The TwiST CK rate constant in HF patients was $0.20 \pm 0.06 \mathrm{~s}^{-1}$ (mean $\pm \mathrm{SD}$ ), significantly lower than that in the healthy group at $0.33 \pm$ $0.08 \mathrm{~s}^{-1}(p=0.00001)$. Results of the previously published TRiST analysis without Q-correction are compared to the ones with $\mathrm{Q}$-correction in an additional figure [see Additional file 1].

\section{Discussion}

We present a new, faster method called TwiST for measuring the forward $\mathrm{CK}$ rate-constant in human heart. The method is validated by Bloch equation analysis and by 

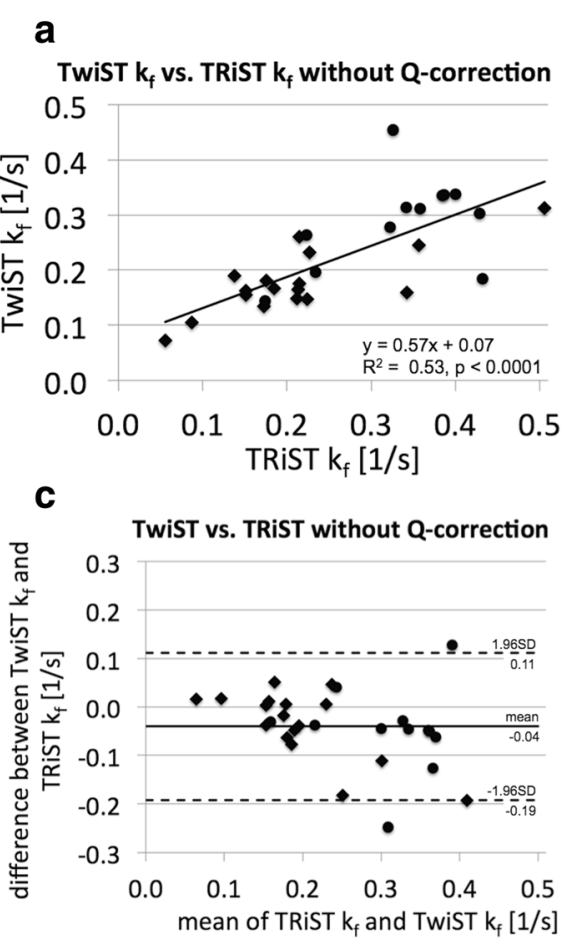

b

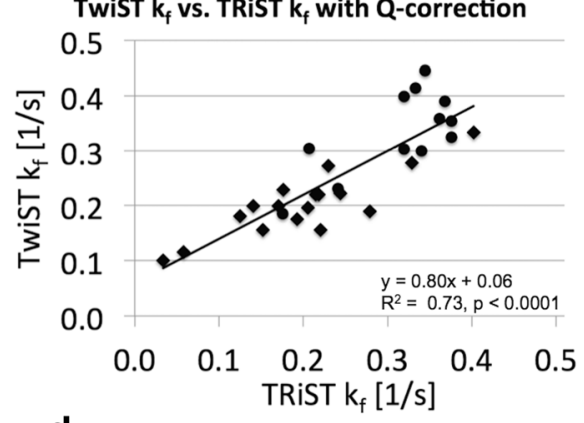

d

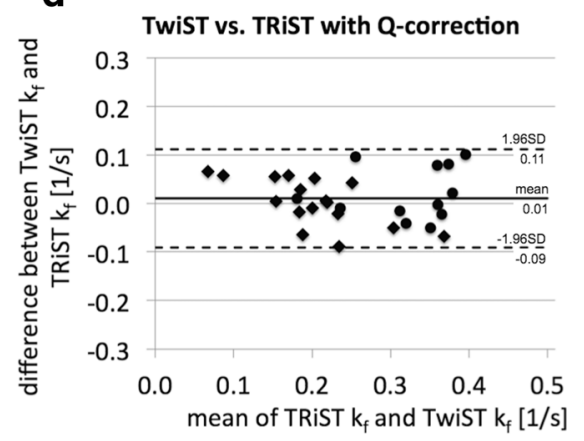

Fig. 7 a, b Linear correlation and (c, d) Bland-Altman plots for TRiST and TwiST cardiac CK pseudo-first-order rate constant $k_{f}$ determined $(\mathbf{a}, \mathbf{c})$ without and $(\mathbf{b}, \mathbf{c})$ with spill-over Q-correction. Data from all 29 participants are shown, dots for healthy participants and diamonds for HF patients

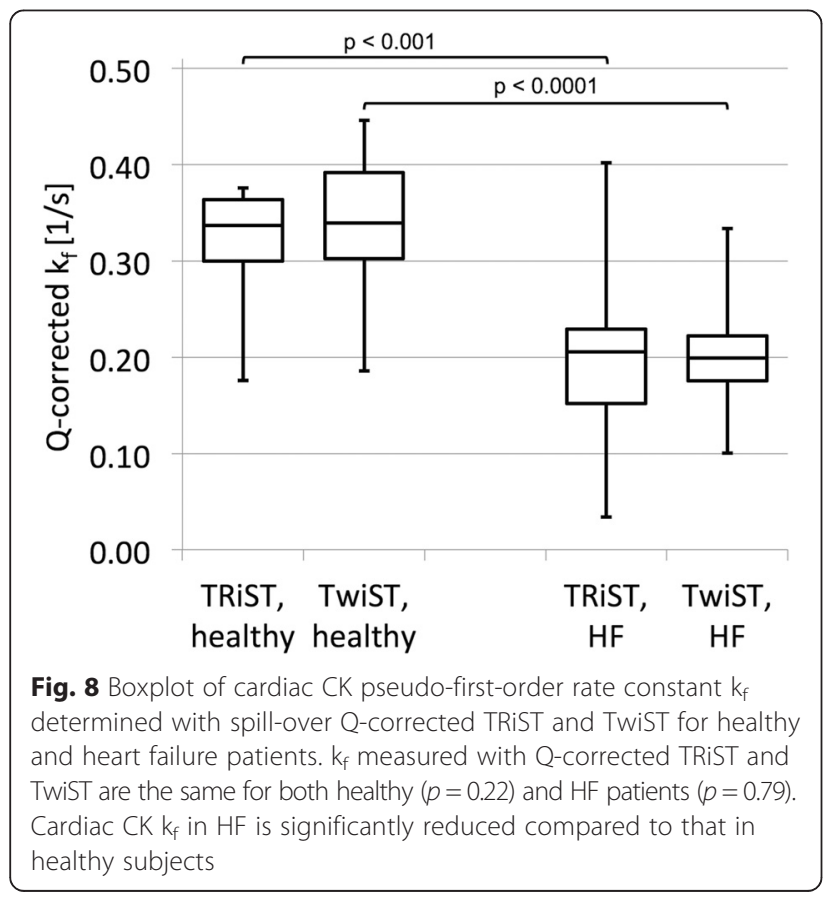

comparison with the previously validated TRiST method in ${ }^{31} \mathrm{P}$ MRS studies of healthy and failing human hearts performed at $3 \mathrm{~T}$. The TwiST method is faster than the TRiST method, requiring one less acquisition and saving $9 \mathrm{~min}$ from the present protocol, or a $23 \%$ efficiency improvement vs. the three TRiST acquisitions. The number of acquisitions required for measuring CK reaction rates has thus now been reduced from four [3] or three [7] to two, resulting in proportionate improvements in efficiency for the ST portion of the protocol. Although the timesaving is not large relative to the entire protocol, it does shorten a long exam, making it more tolerable for patients with cardiovascular disease without introducing significant error.

This study also presented the first $3 \mathrm{~T}$ measurements of cardiac CK kinetics in patients with heart failure. The results show significant reductions in cardiac CK reactionrates that are in quantitative agreement (both mean values and errors) with prior measurements obtained at $1.5 \mathrm{~T}$, where $\mathrm{k}_{\mathrm{f}}$ was $0.21 \pm 0.07 \mathrm{~s}^{-1}$ in HF patients compared to $0.32 \pm 0.07 \mathrm{~s}^{-1}$ in healthy subjects [3]. The new measurements obtained by both TRiST and TwiST methods and at a different field strength of $3 \mathrm{~T}$, provide further independent evidence that CK energy supply is reduced in the failing human heart. A paired comparison of data acquired by the different methods at $1.5 \mathrm{~T}$ and $3 \mathrm{~T}$ from the same subjects was not performed here, as the 
original $1.5 \mathrm{~T}$ scanner is no longer available. Such studies could help elucidate whether the residual scatter has biologic or instrumental origins.

The Monte Carlo simulations show that the expected scatter for a given SNR decreases to $8.3 \%$ in TwiST measurements compared to $13.4 \%$ with TRiST. This is because in TwiST a $T_{1}^{\text {intrinsic }}$, or a range of $\mathrm{T}_{1}^{\text {intrinsic }}$ for the Q-corrected TwiST, is assumed instead of measuring $\mathrm{T}_{1}$ `. In TRiST, $\mathrm{T}_{1}$ is determined from two measurements: $\mathrm{M}^{\prime}\left(\mathrm{TR}_{\text {long }}\right)$ and $\mathrm{M}^{\prime}\left(\mathrm{TR}_{\text {short }}\right)$. Compared to TRiST, TwiST does not measure $M^{\prime}\left(\mathrm{TR}_{\text {short }}\right)$. $\mathrm{M}^{\prime}\left(\mathrm{TR}_{\text {short }}\right)$ is the acquisition with the lowest $\mathrm{PCr}$ signal in TRiST because of the short TR and its chemical exchange with the saturated $\gamma$-ATP. The combination of low signal for $\mathrm{M}^{\prime}\left(\mathrm{TR}_{\text {short }}\right)$ and the inherently low SNR in clinical cardiac ${ }^{31} \mathrm{P}$ MRS settings, makes the determination of $\mathrm{T}_{1}{ }^{\circ}$ critical to the accuracy of TRiST $\mathrm{k}_{\mathrm{f}}$ determinations.

Bloch equation simulations showed that $\mathrm{T}_{1}^{\text {intrinsic }}$ measured with the TRiST sequence underestimates the true value to an extent that depends strongly on the spillover ratio $\mathrm{Q}$. This can confound its determination. For example, assuming an input $T_{1}^{\text {intrinsic }}$ of $7.9 \mathrm{~s}$, the simulations predict apparent $T_{1}^{\text {intrinsic }}$ values of 4 to $7 \mathrm{~s}$ as $\mathrm{Q}$ varies from 0.5 to 1 (Fig. $4 \mathrm{a}$ and b). In the present study, the measured $\mathrm{T}_{1}^{\text {intrinsic }}$ varied from 3.4 to $10.7 \mathrm{~s}$. We therefore assumed a range in the actual intrinsic $T_{1}$ from 6.5 to $9.5 \mathrm{~s}$ for computing the Q-corrected $\mathrm{T}_{1}^{\text {intrinsic }}$ and TRiST/TwiST $k_{f}$ formulae. For the Monte Carlo simulations without spill-over corrections, $\mathrm{T}_{1}^{\text {intrinsic }}=7 \mathrm{~s}$ was chosen to be consistent with simulations performed in [7], and to enable a comparison of the findings. In the present study, there were no significant differences in $\mathrm{T}_{1}^{\text {intrinsic }}$ between healthy subjects and HF patients, whether calculated with or without Q-corrections (Fig. 6). This suggests that the same $T_{1}^{\text {intrinsic }}$ can be assumed for TwiST studies of $\mathrm{k}_{\mathrm{f}}$ in HF patients and healthy subjects. The overall average value pooling the HF patients and healthy subjects was $8.4 \pm 1.4 \mathrm{~s}$. That $\mathrm{T}_{1}^{\text {intrinsic }}$ is the same, is also consistent with the notion that $\mathrm{T}_{1}^{\text {intrinsic }}$ for $\mathrm{PCr}$ is a measure of $\mathrm{T}_{1}$ independent of any exchange effects or differences therein in healthy and HF populations.

The proposed formula for spill-over corrected TwiST, Eq. [8], does not explicitly include $T_{1}^{\text {intrinsic }}$. Nevertheless, the coefficients in Table 2 depend on the range of $\mathrm{T}_{1}^{\text {intrinsic }}$ assumed for their determination. For $\mathrm{Q}=1$, Eq. 8 can be transformed into an equation similar to Eq. 5 ,

$$
k_{f}^{Q-T w i S T}(Q=1)=\frac{1}{8.13}\left(\frac{M_{0}^{\text {control }}}{M^{\prime}\left(T R_{\text {long }}\right)}-1.03\right),
$$

with an equivalent $T_{1}^{\text {intrinsic }}$ of $8.13 \mathrm{~s}$ very close to the value measured in this study. Hence, $T_{1}^{\text {intrinsic }}$ is absorbed into coefficients $l$ and $n$ of Eq. 8. The effect of varying
$\mathrm{T}_{1}^{\text {intrinsic }}$ on $\mathrm{k}_{\mathrm{f}}^{\mathrm{Q}-\mathrm{TwiST}}$ can be determined from Fig. 5 . Apart from this, the coefficients in Table 2 for the spill-over corrected formulae for $\mathrm{T}_{1}^{\mathrm{Q}-\text { intrinsic }}, \mathrm{k}_{\mathrm{f}}^{\mathrm{Q}-\mathrm{TR} i S T}$ and $\mathrm{k}_{\mathrm{f}}^{\mathrm{Q}-\mathrm{TwiST}}$ are only applicable for data acquired with the sequence parameters used in the present study to measure cardiac CK exchange rates at $3 \mathrm{~T}$ with the expected parameter range as given in Table 1. Deviations would in general require determination of a new set of coefficients based on adapted simulations.

The Bloch equation simulation results in Fig. 4 suggest that for $\mathrm{Q}>0.95$ spill-over effects lead to a dip of $\mathrm{T}_{1}^{\text {intrinsic }}$ and both the TRiST and TwiST $k_{\mathrm{f}}$ measurements. $\mathrm{Q}$ values larger than 0.95 only occur for very low saturation power and the dip in $\mathrm{T}_{1}^{\text {intrinsic }}$ and TRiST/TwiST $\mathrm{k}_{\mathrm{f}}$ for $\mathrm{Q}$ values larger than 0.95 is caused by incomplete $\gamma$-ATP saturation. In practice, $Q$ values larger than 0.95 can occur because of low saturation power (leading to both reduced spill-over saturation of $\mathrm{PCr}$ and incomplete $\gamma$-ATP resonance saturation) or because of noise in the acquired spectra. The former can be assessed in the spectra by noting any residual $\gamma$-ATP resonance. We attributed $Q>1$ to noise and rounded $Q$ to 1 in the Q-corrected formulae. Based on Eq. [3], the error in $Q$ is the root of the sum of the squared errors in $M_{O}$ and $M_{O}^{\text {control }}$. In the determination of the Q-corrected formulae the range of saturation power was limited to keep Q below 0.96 to ensure that the dip was not included in the fitting coefficients.

Recently, Bashir et al. presented a time-dependent ST approach to measure CK $\mathrm{k}_{\mathrm{f}}$ values in the human heart at 3 Tesla [24]. Their reported $\mathrm{k}_{\mathrm{f}}=0.32 \pm 0.05 \mathrm{~s}^{-1}$ agrees well with $\mathrm{k}_{\mathrm{f}}$ values of the present work, whereas their $\mathrm{PCr} \mathrm{T}_{1}^{\text {intrinsic }}=7.36 \pm 1.79 \mathrm{~s}$ is somewhat smaller than the Q-corrected $\quad \mathrm{T}_{1}^{\mathrm{Q} \text {-intrinsic }}=8.4 \pm 1.4 \quad \mathrm{~s}$ presented here. Xiong et al. published a very fast ST method applied to in vivo swine hearts at ultra-high field strength (4.7 $\mathrm{T}$ 9.4 T) $[25,26]$. Their fastest $1 \mathrm{D}$ CSI localized $\mathrm{T}_{1}^{\text {nom }}$ method acquires the ST protocols in less than $14 \mathrm{~min}$. This compares to $\sim 40 \mathrm{~min}$ for our Q-corrected TwiST protocol that includes a third acquisition for measuring metabolite concentrations and $\mathrm{Q}$. The $\mathrm{T}_{1}^{\mathrm{nom}}$ method has yet to be translated to human heart studies or combined with concentration measurements. Also it is not compensated for spillover which may be more problematic at lower fields where chemical shift dispersions are proportionately smaller.

\section{Limitations}

Test re-test reproducibility of these methods remains to be studied in the future. The transmit/receive coil and pulse sequences used in this study have been specially designed and built by our research team for cardiac ${ }^{31} \mathrm{P}$ MRS. 


\section{Conclusions}

In conclusion, the spill-over Q-corrected TwiST method can be used to measure the CK pseudo-first-order rateconstant $\mathrm{k}_{\mathrm{f}}$ in the human heart at 3 Tesla with one fewer acquisition compared to the previously presented TRiST method. Instead, a range of $\mathrm{PCr} \mathrm{T}_{1}^{\text {intrinsic }}$ is assumed. It is shown that $\mathrm{T}_{1}^{\text {intrinsic }}$ is the same in healthy subjects and in heart failure patients. The values of $\mathrm{k}_{\mathrm{f}}$ measured with Q-corrected TwiST closely agree with earlier measurements at 1.5 Tesla, and demonstrate a significant reduction in failing, compared to healthy hearts.

\section{Additional file}

Additional file 1: Boxplot of cardiac CK pseudo-first-order rate constant $\mathbf{k}_{\mathrm{f}}$ determined with TRiST, spill-over Q-corrected TRiST, and spill-over Q-corrected TwiST for healthy and heart failure patients. $\mathrm{k}_{\mathrm{f}}$ measured with TRiST, with Q-corrected TRiST, and with Q-corrected TwiST are the same in both healthy and HF patients. Cardiac CK $\mathrm{k}_{\mathrm{f}}$ in $\mathrm{HF}$ is significantly reduced compared to that in normal subjects. (PDF $60 \mathrm{~kb}$ )

\section{Abbreviations}

1D CSI: One-dimensional chemical shift imaging; ${ }^{31} \mathrm{P}$ : Phosphorus; ATP: Adenosine triphosphate; CK: Creatine kinase; DANTE: Delays alternating with nutations for tailored excitation; HF: Heart failure; PCr: Creatine phosphate; Q: Spill over ratio, see equation 3; SD: Standard deviation; ST: Saturation transfer; TRiST: Triple Repetition time Saturation Transfer; TwiST: Two Repetition time Saturation Transfer.

\section{Competing interests}

MS was an employee of Philips Healthcare until May 2014, the manufacturer of equipment used in this study.

\section{Authors' contributions}

MS made substantial contributions to conception and design, the acquisition, analysis and interpretation of data; performed part of the simulations, and drafted and revised the manuscript. RG made substantial contributions to interpretation of data; performed part of the simulations, and critically revised the manuscript for important intellectual content. AE made substantial contributions to conception and design, the acquisition and interpretation of data, and critically revised the manuscript for important intellectual content. AS made substantial contributions to the acquisition of data, and critically revised the manuscript for important intellectual content. $\mathrm{PB}$ made substantial contributions to conception and design, the interpretation of data, and critically revised the manuscript for important intellectual content. RW made substantial contributions to conception and design, the acquisition and interpretation of data, and critically revised the manuscript for important intellectual content. All authors read and approved the final manuscript.

\section{Acknowledgements}

NIH: R01 HL56882, R01 HL61912, R01 HL63030; AHA grant \#13GRNT17050100

\begin{abstract}
Author details
${ }^{1}$ Division of MR Research, Russell H. Morgan Department of Radiology and Radiological Science, The Johns Hopkins University School of Medicine, Baltimore, MD, USA. ${ }^{2}$ Department of Diagnostic and Interventional Imaging, University of Texas Health Science Center at Houston, Houston, TX, USA. ${ }^{3}$ Systems and Biomedical Engineering Department, Faculty of Engineering, Cairo University, Giza, Egypt. ${ }^{4}$ Cardiology Division, Department of Medicine, The Johns Hopkins University School of Medicine, Baltimore, MD, USA.
\end{abstract}

Received: 18 February 2015 Accepted: 21 July 2015

Published online: 08 August 2015
References

1. Forsén S, Hoffman RA. Study of moderately rapid chemical exchange reactions by means of nuclear magnetic double resonance. J Chem Phys. 1963;39:2892.

2. Brown TR, Gadian DG, Garlick PB, Radda GK, Seeley PJ, Styles P. Creatine kinase activities in skeletal and cardiac muscle measured by saturation transfer NMR. Front Biol Energ. 1978;2:1341-9.

3. Bottomley PA, Ouwerkerk R, Lee RF, Weiss RG. Four-angle saturation transfer (FAST) method for measuring creatine kinase reaction rates in vivo. Magn Reson Med. 2002:47:850-63.

4. Weiss RG, Gerstenblith G, Bottomley PA. ATP flux through creatine kinase in the normal, stressed, and failing human heart. Proc Natl Acad Sci U S A. 2005;102:808-13.

5. Smith CS, Bottomley PA, Schulman SP, Gerstenblith G, Weiss RG. Altered Creatine Kinase Adenosine Triphosphate Kinetics in Failing Hypertrophied Human Myocardium. Circulation. 2006;114:1151-8.

6. Bottomley PA, Panjrath GS, Lai S, Hirsch GA, Wu K, Najjar SS, et al. Metabolic Rates of ATP Transfer Through Creatine Kinase (CK Flux) Predict Clinical Heart Failure Events and Death. Sci Transl Med. 2013;5:215re3-3.

7. Schär M, El-Sharkawy A-MM, Weiss RG, Bottomley PA. Triple repetition time saturation transfer (TRiST) 31P spectroscopy for measuring human creatine kinase reaction kinetics. Magn Reson Med. 2010;63:1493-501.

8. El-Sharkawy A-M, Schär M, Ouwerkerk R, Weiss RG, Bottomley PA. Quantitative cardiac ${ }^{31} \mathrm{P}$ spectroscopy at 3 Tesla using adiabatic pulses. Magn Reson Med. 2009;61:785-95.

9. Gabr RE, Weiss RG, Bottomley PA. Correcting reaction rates measured by saturation-transfer magnetic resonance spectroscopy. J Magn Reson. 2008;191:248-58.

10. Kingsley PB, Monahan WG. Corrections for off-resonance effects and incomplete saturation in conventional (two-site) saturation-transfer kinetic measurements. Magn Reson Med. 2000;43:810-9.

11. Rees D, Smith MB, Harley J, Radda GK. In vivo functioning of creatine phosphokinase in human forearm muscle, studied by 31P NMR saturation transfer. Magn Reson Med. 1989;9:39-52.

12. Friedrich J, Nascimben $L$, Liao R, Ingwall JS. Phosphocreatine $T 1$ measurements with and without exchange in the heart. Magn Reson Med. 1993:30:45-50

13. Schär M, El-Sharkawy A-MM, Bottomley PA, Weiss RG. Reduced myocardial creatine kinase reaction rates in human heart failure: first measurements at 3T. In: Proceedings of the Joint Annual Meeting ISMRM-ESMRMB 2010. Stockholm, Sweden. 2010. p. 166.

14. El-Sharkawy A-MM, Gabr RE, Schär M, Weiss RG, Bottomley PA. Quantification of human high-energy phosphate metabolite concentrations at 3 T with partial volume and sensitivity corrections. NMR Biomed. 2013;26:1363-71

15. Yabe T, Mitsunami K, Inubushi T, Kinoshita M. Quantitative measurements of cardiac phosphorus metabolites in coronary artery disease by 31P magnetic resonance spectroscopy. Circulation. 1995;92:15-23.

16. Bottomley PA, Wu KC, Gerstenblith G, Schulman SP, Steinberg A, Weiss RG. Reduced myocardial creatine kinase flux in human myocardial infarction: an in vivo phosphorus magnetic resonance spectroscopy study. Circulation. 2009;119:1918-24.

17. Beer M, Seyfarth T, Sandstede J, Landschütz W, Lipke C, Köstler H, et al. Absolute concentrations of high-energy phosphate metabolites in normal, hypertrophied, and failing human myocardium measured noninvasively with (31)P-SLOOP magnetic resonance spectroscopy. J Am Coll Cardiol. 2002;40:1267-74

18. Machann W, Breunig F, Weidemann F, Sandstede J, Hahn D, Köstler H, et al. Cardiac energy metabolism is disturbed in Fabry disease and improves with enzyme replacement therapy using recombinant human galactosidase A. Eur J Heart Fail. 2011;13:278-83.

19. Spencer RG, Ferretti JA, Weiss GH. NMR saturation factors in the presence of chemical exchange. J Magn Reson. 1989:84:223-35.

20. McConnell HM. Reaction rates by nuclear magnetic resonance. J Chem Phys. 1958:28:430.

21. Zwart NR, Pipe JG. Graphical programming interface: A development environment for MRI methods: Graphical Programming Interface. Magn. Reson. Med. 2014:n/a-n/a. 10.1002/mrm.25528.

22. Meyerspeer M, Krssak M, Moser E. Relaxation times of $31 \mathrm{P}$-metabolites in human calf muscle at 3 T. Magn Reson Med. 2003;49:620-5. 
23. Schär M, Kozerke S, Fischer SE, Boesiger P. Cardiac SSFP imaging at 3 Tesla. Magn Reson Med. 2004;51:799-806.

24. Bashir A, Gropler R. Reproducibility of creatine kinase reaction kinetics in human heart: a ${ }^{31} \mathrm{P}$ time-dependent saturation transfer spectroscopy study. NMR Biomed. 2014;27:663-71.

25. Xiong Q, Li Q, Mansoor A, Jameel MN, Du F, Chen W, et al. Novel strategy for measuring creatine kinase reaction rate in the in vivo heart. AJP Heart Circ Physiol. 2009:297:H1010-9.

26. Xiong Q, Du F, Zhu X, Zhang P, Suntharalingam P, Ippolito J, et al. ATP production rate via Creatine Kinase or ATP synthase in vivo: a novel superfast magnetization saturation transfer method. Circ Res. 2011;108:653-63.

\section{Submit your next manuscript to BioMed Central} and take full advantage of:

- Convenient online submission

- Thorough peer review

- No space constraints or color figure charges

- Immediate publication on acceptance

- Inclusion in PubMed, CAS, Scopus and Google Scholar

- Research which is freely available for redistribution 